\title{
Sicherheitsmanagement der Therapie mit Antimalariamitteln: was bedeuten die neuen Empfehlungen für die Praxis?
}

\section{Safety Management in Therapy with Antimalarial Medication: What do the New Recommendations Mean for Clinical Practice?}

Autoren

Denitsa Hadjiski, Christoph Fiehn

Institut

Tätigkeitsschwerpunkt Klinische Immunologie, Rheumatologie Baden-Baden GbR, Baden-Baden

Schlüsselwörter

Hydroxychloroquin, Chloroquin, Antimalariamittel, Retinopathie, systemischer Lupus erythematodes

Key words

chloroquine, antimalarial, retinopathy, systemic lupus erythematosus, hydroxychloroquine

Bibliografie

DOI https://doi.org/10.1055/a-1185-8566

Online-Publikation: 16.7.2020

Akt Rheumatol 2020; 45: 314-318

(c) Georg Thieme Verlag KG Stuttgart · New York ISSN 0341-051X

Korrespondenzadresse

Dr. Denitsa Hadjiski

Rheumatologie Baden-Baden GbR

Tätigkeitsschwerpunkt Klinische Immunologie

Beethovenstraße 2

76530 Baden-Baden

Tel.: 07221302800, Fax: 072213028020

d.hadjiski@rheuma-badenbaden.de

\section{ZUSAMMENFASSUNG}

Antimalariamittel (AM) haben nach wie vor eine große Bedeutung in der Rheumatologie. Die aktualisierten Empfehlungen zum Sicherheitsmanagement mit AM wurden auf der Basis einer systematischen Literaturaturrecherche und im interdisziplinären Konsens erarbeitet. Verantwortlich war die Kommission Pharmakotherapie der Deutschen Gesellschaft für Rheumatologie. Um Nebenwirkungen frühzeitig zu entdecken, bzw. Komplikationen im Zusammenhang mit der AM-Therapie vorzubeugen, sind folgende Maßnahmen empfohlen: Die rheumatologische Therapie mit AM sollte mit Hydroxychloroquin (HCQ) erfolgen und die Dosis von 5 mg/kg Körpergewicht (KG) nicht übersteigen. In den ersten 6 Monaten der Therapie ist eine augenärztliche Untersuchung empfohlen. Risikofaktoren für die Entwicklung einer AM-induzierten Retinopathie sind vorbestehende Makulopathie, Niereninsuffizienz mit glomerulärer Filtrationsrate (GFR) $<60 \mathrm{ml} / \mathrm{min}$, Tamoxifen-Begleittherapie, Tagesdosen HCQ > $5 \mathrm{mg} / \mathrm{kg}$ KG, sowie die Therapie mit Chloroquin (CQ). Bei Vorliegen von Risikofaktoren werden die weiteren Kontrolluntersuchungen einmal pro Jahr empfohlen, ansonsten erst ab dem fünften Jahr, dann ebenfalls jährlich. Die ophthalmologische Untersuchung sollte mindestens eine subjektive und eine objektive Methode beinhalten. Empfohlen wird jeweils das automatisierte Gesichtsfeld (aGF) und die optische Kohärenztomografie (OCT). In der aGF lassen bei einer AMRetinopathie eine parafoveale Empfindlichkeitsabnahme und in der OCT eine umschriebene Verdünnung der Photorezeptorenschicht parafoveal sowie fokale Unterbrechung der Außensegmentstrukturlinie den Verdacht auf eine AM-Retinopathie zu. Zur Erkennung von Kardiomyopathie bzw. Myopathie wird zu Beginn und dann alle 3-6 Monate die Bestimmung von Creatinkinase (CK) und Laktatdehydrogenase (LDH) im Serum empfohlen. Ergänzend können bei Verdacht auf AM-induzierte Kardiomyopathie Troponin, brain natriuretic peptide (BNP), Elektrokardiogramm (EKG), sowie eine kardiale Magnetresonanztomografie (MRT) erwogen werden. Bei Verdacht auf AM-induzierte Myopathie ist die Muskelbiopsie mit elektronenmikroskopischer Untersuchung der Goldstandard. Die Behandlung mit HCQ verursacht bei Einnahme in der Schwangerschaft keine Fehlbildungen der ungeborenen Kinder und ist sowohl in der Schwangerschaft wie auch Stillzeit nach gegenwärtigem Wissenstand sicher. Die aktualisierten Empfehlungen zum Sicherheitsmanagement der Therapie mit AM enthalten strengere Dosisvorgaben, Definition für Risikofaktoren für AMRetinopathie und geeignete ophthalmologische Untersuchungen, sowie Stratifizierung im Monitoring.

\section{ABSTRACT}

Antimalarial medication (AM) continues to play an important role in rheumatology. The updated consensus-based recommendations for safety management with AM were developed on the basis of a systematic literature review in an interdisciplinary consensus. The project was initiated by the Commission 
on Pharmacotherapy of the German Society for Rheumatology. To detect early side-effects and prevent complications in therapy with $A M$, the following precautions need to be taken: Therapy with AM from a rheumatological viewpoint should be conducted with hydroxychloroquine (HCQ) and the dose should not exceed $5 \mathrm{mg} / \mathrm{kg} /$ body weight per day. An ophthalmological examination is recommended in the first 6 months of therapy. Risk factors for the development of AM-induced retinopathy include pre-existing retinopathy, renal failure with a glomerular filtration rate (GFR) of $<60 \mathrm{ml} / \mathrm{min}$, tamoxifen co-medication, daily doses of HCQ $>5 \mathrm{mg} / \mathrm{kg}$ body weight, and treatment with chloroquine (CQ). If risk factors are present, further annual check-ups should be arranged once a year, otherwise annually after 5 years of therapy. The ophthalmological examination should include at least one subjective and one objective method. An automated visual field test (aGF) and optical coherence tomography (OCT) are the most suitable ones. In case of AM retinopathy, the aGF test would show a parafoveal decrease in sensitivity, while the OCT test would reveal a circumscribed thinning of the photoreceptor layer in the parafoveal area and focal interruption of the outer segment structure line. Determination of serum creatine kinase (CK) and lactate dehydrogenase (LDH) is recommended at the beginning of treatment and should be repeated every 3-6 months in order to detect AM-induced cardiomyopathy or myopathy. In case of suspected cardiomyopathy, serum troponin and brain natriuretic peptide (BNP), an electrocardiogram (ECG) and cardiac magnetic resonance imaging (MRI) should be considered in addition. If AM-induced myopathy is suspected, the gold standard is muscle biopsy with electron microscopy. Taking HCQ does not cause malformations in unborn children and is safe during pregnancy and lactation according to current knowledge. The updated recommendations on AM therapy imply stricter dose specifications, a definition of risk factors for AM retinopathy and suitable ophthalmological examinations as well as stratification in monitoring.

\section{Einführung}

Antimalariamittel (AM), insbesondere Hydroxychloroquin (HCQ) spielen eine wichtige Rolle in der Therapie des systemischen Lupus erythematodes (SLE) [1]. Aber auch beim Sjögren-Syndrom [2] und dem Anti-Phospholipid-Syndrom [3] kann nach den derzeit gültigen Empfehlungen HCQ erwogen werden, wenn es in diesen Indikationen auch in Deutschland dafür nicht zugelassen ist. HCQ gehört als Kombinationspartner im sogenannten O’Dell-Schema zusammen mit Methotrexat und Sulfasalazin zu den möglichen Therapieoptionen in der Therapie der rheumatoiden Arthritis [4]. Die Verträglichkeit von HCQ ist in der Regel sehr gut. Unerwünschte Nebenwirkungen wie die gefürchtete Retinopathie aber auch die sehr seltenen Myopathien und Kardiomyopathien wurden berichtet. Die AM-induzierte Retinopathie ist eine seltene Komplikation, kann aber unerkannt zur Erblindung führen. Daher ist ein zielgerichtetes Vorgehen im Sicherheitsmanagement der Therapie mit AM wichtig, um eine frühzeitige Erkennung von Nebenwirkungen bei gleichzeitig praktikablem Aufwand im Praxisalltag zu ermöglichen.

\section{Methoden}

Als Grundlage der aktualisierten Empfehlung zum Sicherheitsmanagement der Therapie mit AM diente die systematische Literaturrecherche in den Datenbanken PubMed und Cochrane, bei der über 1160 Arbeiten zur Sicherheit der Therapie mit AM in der Rheumatologie identifiziert wurden. Manuell wurden zusätzlich als wichtig eingestufte Artikel hinzugefügt. Daraus wurden 67 besonders relevante Publikationen selektiert und genauer analysiert. Diese dienen als Grundlage für die konsensbasierten Empfehlungen der Kommission Pharmakotherapie der Deutschen Gesellschaft für Rheumatologie [5].

\section{Ergebnisse}

\section{Auswahl und Dosis der AM-Therapie}

Alle Patienten, welche wegen einer rheumatologischen Indikation mit AM behandelt werden, sollten HCQ erhalten. Die Therapie mit Chloroquin (CQ) wird wegen der höheren Nebenwirkungsrate nicht empfohlen [6, 7]. Die Dosis sollte individuell errechnet werden und $5 \mathrm{mg} / \mathrm{kg}$ Körpergewicht (KG) pro Tag nicht übersteigen. Das Idealgewicht kommt nicht zur Anwendung. Bei Niereninsuffizienz sollte bei einer glomerulären Filtrationsrate (GFR) $<30 \mathrm{ml} / \mathrm{min}$ eine Dosisanpassung auf max. $3 \mathrm{mg} / \mathrm{kg}$ KG Tag erfolgen.

\section{Risikostratifizierte Screeninguntersuchungen}

Wichtig ist es schon zu Beginn Patienten mit einem erhöhten Risiko für die Entwicklung einer AM-induzierten Retinopathie zu erkennen. Zu den Risikofaktoren gehören eine vorbestehende Makulopathie mit signifikantem zentralen Schaden der Photorezeptorenschicht, renale Insuffizienz mit GFR $<60 \mathrm{ml} / \mathrm{min}$, TamoxifenBegleittherapie, Tagesdosen $>5 \mathrm{mg} / \mathrm{kg} \mathrm{KG}$, sowie die Therapie mit CQ statt HCQ [6-9]. In den ersten 6 Monaten der Therapie sollte eine augenärztliche Basisuntersuchung erfolgen. Ziel dieser Untersuchung ist es, eine vorbestehende Makulopathie zu erkennen. Bei Patienten mit erhöhtem Risiko für die Entwicklung einer Retinopathie sollten die augenärztlichen Untersuchungen von Anfang an jährlich erfolgen. Bei Patienten ohne Risikofaktoren sind die jährlichen Kontrolluntersuchungen erst ab 5 Jahren Einnahmedauer empfohlen ( $>$ Abb. 1). Das Risiko der Entwicklung einer AM-induzierten Retinopathie ist von der Dosis und Dauer der Einnahme des Medikaments abhängig [10-12].

\section{Empfohlene ophthalmologische Untersuchungsmethoden}

Sowohl die Basisuntersuchung, als auch die Kontrollen sollten mindestens eine geeignete subjektive und eine objektive Methode nutzen, i.d.R. sind dies das automatisierte Gesichtsfeld (aGF) und die 


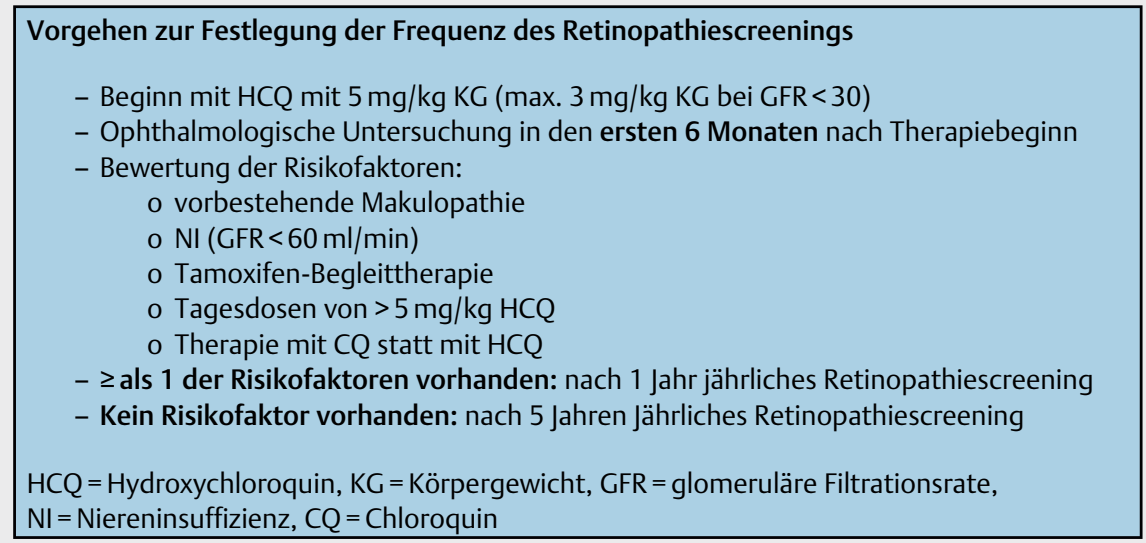

Abb. 1 Vorgehen zur Festlegung der Frequenz des Retinopathiescreenings.

-Tab. 1 Empfohlene ophthalmologische Untersuchungsmethoden zum Retinopathiescreening unter AM-Therapie und Befunde, welche den V.a. eine AM-Retinopathie zulassen.

\begin{tabular}{|l|l|}
\hline Methode & Befunde mit V.a. AM-Retinopathie \\
\hline Subjektive Methode & \\
\hline aGF & parafoveale Empfindlichkeitsabnahme \\
\hline Objektive Methoden & \\
\hline OCT & $\begin{array}{l}\text { umschriebene Verdünnung der Photorezep- } \\
\text { torschicht parafoveal }\end{array}$ \\
\hline & $\begin{array}{l}\text { fokale Unterbrechungen der Außenseg- } \\
\text { mentstrukturlinie }\end{array}$ \\
\hline FAF & $\begin{array}{l}\text { lokale parafoveale erhöhte Autofluoreszenz } \\
\text { (frühe Veränderung) }\end{array}$ \\
\hline $\begin{array}{l}\text { verminderte Autofluoreszenz } \\
\text { (späte Veränderung) }\end{array}$ \\
\hline mf-ERG & parafoveale Abnahme der Rezeptorantwort \\
\hline $\begin{array}{l}\text { aGF= automatisierte Gesichtsfeld, OCT= optische Kohärenztomo- } \\
\text { grafie, FAF = Fundus-Autofluoreszenz, mf-ERG = multifokale }\end{array}$ \\
\hline Elektroretinografie.
\end{tabular}

optische Kohärenztomografie (OCT) [13-23]. Die aGF sollte standardmäßig mit 10 Grad Durchmesser, 2 Grad Prüfabstand = 10-2 durchgeführt werden, bei asiatischen Patienten sind z.T. andere Prüfbedingungen (24-2 oder 30-2) erforderlich. Das aGF hat als subjektiver Test eine gewisse Variabilität, daher sollte der Test bei erstmaliger Messung eines Defektes wiederholt werden.

Weitere geeignete objektive Methoden sind die multifokale Elektroretinografie (mf-ERG) oder die Fundus-Autofluoreszenz (FAF) $[14,17]$. In $>$ Tab. 1 sind die wichtigsten objektiven und subjektiven Methoden, sowie die pathologischen Befunde, die den Verdacht auf eine AM-Retinopathie zulassen, zusammengefasst. ( $\triangleright$ Tab. 2 zeigt die interdisziplinären Empfehlungen zum Sicherheitsmanagement der Therapie mit AM zusammengefasst.)

\section{Leitsymptome und Manifestationen der seltenen Myo- und Kardiomyopathie}

Vor Beginn der Therapie und alle 3-6 Monaten sollten Creatinkinase (CK) und Laktatdehydrogenase (LDH) im Blut bestimmt werden, um eine Myopathie bzw. Kardiomyopathie möglichst frühzeitig zu erkennen. Am häufigsten werden von Patienten Muskelschwäche oder Myalgien als Symptome der Myopathie angegeben. Bei Erhöhung der CK und LDH sollte eine Elektromyografie (EMG) angeschlossen und bei Nachweis von einem myopathischen Muster eine Muskelbiopsie mit Elektronenmikroskopie durchgeführt werden. Bei rechtzeitigem Erkennen der AM-induzierten Myopathie und Absetzen der Therapie waren die Beschwerden in den beschriebenen Fällen vollständig regredient [24].

Die Kardiomyopathie macht sich als Herzinsuffizienz mit den typischen Symptomen wie Dyspnoe, Beinödeme und Husten bemerkbar. Im Labor finden sich Erhöhung von Troponin, CK und brain natriuretic peptide (BNP) [25]. Im Elektrokardiogramm (EKG) sind Reizleitungsstörungen und in der kardialen Magnetresonanztomografie (MRT) spezifische Befunde einer ventrikulären Hypertrophie zu erkennen [25]. In Ausnahmefälle kann eine Herzmuskelbiopsie zur Sicherung der Diagnose mit dem Nachweis der typischen Einlagerungen von Myeloid-Körperchen in der Elektronenmikroskopie notwendig sein. Auch die Kardiomyopathie ist potentiell reversibel (bei 7 von 8 Fällen) [25]. Inwieweit auch isolierte Reizleitungstörungen, die als häufigste kardiale Nebenwirkungen von AM beschrieben sind [26], tatsächlich nachvollziehbar durch AM ausgelöst werden, ist derzeit noch nicht sicher geklärt [27].

\section{Schwangerschaft und Stillzeit}

Es gibt keine Evidenz in der Literatur für vermehrte Malformationen der Augen oder Ohren bei Säugligen von Müttern, die mit HCQ therapiert wurden [28-30]. HCQ ist nach gegenwärtigem Wissensstand in der Schwangerschaft und Stillzeit sicher und kann bei entsprechender Indikation weiter gegeben werden. 
\Tab. 2 Interdisziplinäre Empfehlungen zum Sicherheitsmanagement der Therapie mit Antimalariamitteln in der Rheumatologie.

\begin{tabular}{|c|c|c|}
\hline Nr. & Empfehlungen & $\begin{array}{l}\text { Empfeh- } \\
\text { lungsgrad* }\end{array}$ \\
\hline 1 & $\begin{array}{l}\text { Eine Therapie mit Antimalariamittel (AM) in der Rheumatologie sollte mit Hydroxychloroquin (HCQ) erfolgen und die Dosis von } 5 \\
\mathrm{mg} / \mathrm{kg} \text { Körpergewicht (KG) pro Tag nicht überschreiten. }\end{array}$ & B \\
\hline 2 & In den ersten 6 Monaten der Therapie mit AM sollte eine augenärztliche Basisuntersuchung erfolgen. & B \\
\hline 3 & $\begin{array}{l}\text { Eine vorbestehende Makulopathie, Niereninsuffizienz (GFR }<60 \mathrm{ml} / \mathrm{min} \text { ), Tamoxifen-Begleittherapie, Tagesdosen von }>5 \mathrm{mg} / \mathrm{kg} \mathrm{KG} \\
\text { pro Tag HCQ oder eine Therapie mit Chloroquin (CQ) statt mit HCQ stellen ein erhöhtes Risiko für eine AM-induzierte Retinopathie } \\
\text { dar. }\end{array}$ & B \\
\hline 4 & $\begin{array}{l}\text { Patienten mit erhöhtem Retinopathie-Risiko (siehe 3.) sollten von Beginn an jährlich augenärztliche Kontrollen erhalten, während } \\
\text { dies bei Patienten ohne Risikofaktoren erst ab } 5 \text { Jahren Einnahmedauer empfohlen wird. }\end{array}$ & B \\
\hline 5 & $\begin{array}{l}\text { Die Untersuchung (Basisuntersuchung und Kontrollen) sollte mindestens je eine geeignete subjektive und objektive Methode } \\
\text { nutzen, i.d.R. sind dies das automatisierte Gesichtsfeld (aGF) und die optische Kohärenztomografie (OCT). Als objektive Methoden } \\
\text { sind auch die multifokale Elektroretinografie (mf-ERG) oder die Fundus-Autofluoreszenz (FAF) geeignet. }\end{array}$ & B \\
\hline 6 & $\begin{array}{l}\text { Bei dem Retinopathiescreening sollte auf parafoveale Empfindlichkeitsabnahme im aGF und eine umschriebene Verdünnung der } \\
\text { Photorezeptorschicht parafoveal oder fokale Unterbrechungen der Außensegmentstrukturlinie im OCT geachtet werden, da dies } \\
\text { Zeichen für eine AM Retinopathie sind. }\end{array}$ & B \\
\hline 7 & Bei Niereninsuffizienz sollte bei GFR< 30 ml/min eine Dosisanpassung auf max. 3 mg/kg KG/Tag erfolgen. & $\mathrm{D}$ \\
\hline 8 & $\begin{array}{l}\text { Vor Therapie und dann ca. alle 3-6 Monate sollten CK und LDH im Blut bestimmt werden. Dies sind geeignete Screening-Untersu- } \\
\text { chungen zur Frage einer Myopathie oder Kardiomyopathie. Ggf. sind zusätzlich Aldolase im Serum und EMG, bzw. kardiale } \\
\text { Biomarker wie Troponin oder BNP, EKG und kardiale Bildgebung sinnvoll. }\end{array}$ & B \\
\hline 9 & $\begin{array}{l}\text { HCQ ist in der Schwangerschaft und Stillzeit nach gegenwärtigem Wissen sicher und sollte bei entsprechender Indikation } \\
\text { fortgeführt werden. }\end{array}$ & B \\
\hline
\end{tabular}

\section{Diskussion}

Nach systematischer Auswertung der aktuellen Literatur zur Toxizität von HCQ ist festzustellen, dass die AM-induzierten Nebenwirkungen stark von der Dosis und Dauer der Behandlung abhängen [10-12]. Die richtige Wahl des AM spielt ebenfalls eine große Rolle. CQ sollte aufgrund der vermutlich höheren Rate an Retinopathien, Myopathien, sowie Kardiomyopathien nur noch in Ausnahmefälle verwendet werden [6, 7]. Die aktualisierte Dosisempfehlung von max. 5 mg/kg KG für HCQ wird das Risiko für Retinopathien senken.

In Übereinstimmung mit den amerikanischen und britischen Empfehlungen [31, 32] zum ophthalmologischen Screening wurden in den neuen deutschen Empfehlungen [5] je nach Risiko neue Intervalle für die augenärztlichen Untersuchungen definiert. Der Hintergrund ist, dass die AM-induzierte Retinopathie in den ersten 5 Jahren der Einnahme nur selten auftritt [10].

Die Empfehlung für die Verwendung einer subjektiven und einer objektiven Methode sollte die ophthalmologischen Kontrollen vereinheitlichen. Bei fraglichen Befunden ist eine Kontrolle in einem spezialisierten ophthalmologischen Zentrum zu erwägen. Die Entscheidung über das Absetzen der AM-Therapie sollte möglichst im Dialog zwischen Patient, Augenarzt und Rheumatologe getroffen werden.

\section{FAZIT FÜR DIE PRAXIS}

Die Therapie mit HCQ ist sicher und hat das Potenzial, die Mortalität der Patienten mit SLE durch Verhinderung von kardiovaskulären Ereignissen zu reduzieren. Es ist wichtig mögliche Nebenwirkungen frühzeitig zu erkennen, um die Rate an Komplikationen durch die Therapie zu minimieren. Vor allem die strengeren Dosisvorgaben, die Risikostratifizierung im Monitoring und die definierten augenärztlichen Untersuchungen sind neu und werden das Sicherheitsmanagement der AM-Therapie standardisieren und verbessern.

\section{Interessenkonflikt}

Die Autorinnen/Autoren geben an, dass kein Interessenkonflikt besteht. 
Literatur

[1] Fanouriakis A, Kostopoulou M, Alunno A et al. (2019) 2019 update of the EULAR recommendations for the management of systemic lupus erythematosus. Ann Rheum Dis 2019; 78: 736-745

[2] Ramos-Casals M, Brito-Zerón P, Bombardieri S et al. (2019) EULAR recommendations for the management of Sjögren's syndrome with topical and systemic therapies. Ann Rheum Dis 2020; 79: 3-18

[3] Tektonidou MG, Andreoli L, Limper M et al. (2019) EULAR recommendations for the management of antiphospholipid syndrome in adults. Ann Rheum Dis 2019; 78: 1296-1304

[4] Fiehn C, Holle J, Iking-Konert C et al. S2e-Leitlinie: Behandlung der rheumatoiden Arthritis mit krankheitsmodifizierenden Medikamenten. Z Rheumatol 2018; 77 (Suppl 2): 35-53

[5] Fiehn C, Ness T, Weseloh C et al. Sicherheitsmanagement der Therapie mit Antimalariamitteln in der Rheumatologie. Interdisziplinäre Empfehlungen auf der Basis einer systematischen Literatur-Recherche. Z Rheumatol 2020; 79: 186-194

[6] Finbloom DS, Silver K, Newsome DA et al. Comparison of hydroxychloroquine and chloroquine use and the development of retinal toxicity. J Rheumatol 1985; 12: 692-694

[7] Chatrel C, Roubille F, Vernhet $\mathrm{H}$ et al. Cardiac Complications Attributed to Chloroquine and Hydroxychloroquine: A Systematic Review of the Literature. Drug Saf 2018; 41: 919-931

[8] Wolfe F, Marmor MF. Rates and predictors of hydroxychloroquine retinal toxicity in patients with rheumatoid arthritis and systemic lupus erythematosus. Arthritis Care Res 2010; 62: 775-784

[9] Aletaha D, Kapral T, Smolen JS. Toxicity profiles of traditional disease modifying antirheumatic drugs for rheumatoid arthritis. Ann Rheum Dis 2003; 62: 482-486

[10] Melles RB, Marmor MF. The risk of toxic retinopathy in patients on long-term hydroxychloroquine therapy. JAMA Ophthalmol 2014; 132: $1453-1460$

[11] Mavrikakis I, Sfikakis PP, Mavrikakis E et al The incidence of irreversible retinal toxicity in patients treated with hydroxychloroquine: a reappraisal. Ophthalmology 2003; 110: 1321-1326

[12] Mavrikakis M, Papazoglou S, Sfikakis PP et al Retinal toxicity in long term hydroxychloroquine treatment. Ann Rheum Dis 1996; 55: 187-189

[13] Hart WM, Burde RM, Johnston GP et al (1984). Static perimetry in chloroquine retinopathy. Perifoveal patterns of visual field depression. Arch Ophthalmol Chic III 1960; 102: 377-380

[14] Cukras C, Huynh N, Vitale S et al. Subjective and objective screening tests for hydroxychloroquine toxicity. Ophthalmology 2015; 122: 356-366

[15] Rodriguez-Padilla JA, Hedges TR et al. (2007) High-speed ultra-highresolution optical coherence tomography findings in hydroxychloroquine retinopathy. Arch Ophthalmol Chic III 1960; 125: 775-780

[16] RSHM Allam, Abd-Elmohsen MN, Khafagy MM et al. Spectral-Domain Optical Coherence Tomography of Preclinical Chloroquine Maculopathy in Egyptian Rheumatoid Arthritis Patients. J Ophthalmol 2015; 2015: 292357

[17] Ahn SJ, Ryu SJ, Joung JY et al. Choroidal Thinning Associated With Hydroxychloroquine Retinopathy. Am J Ophthalmol 2017; 183: 56-64
[18] De Sisternes L, Hu J, Rubin DL et al. Analysis of Inner and Outer Retinal Thickness in Patients Using Hydroxychloroquine Prior to Development of Retinopathy. JAMA Ophthalmol 2016; 134: 511-519

[19] Allahdina AM, Stetson PF, Vitale S et al. Optical Coherence Tomography Minimum Intensity as an Objective Measure for the Detection of Hydroxychloroquine Toxicity. Invest Ophthalmol Vis Sci 2018; 59: 1953-1963

[20] Maturi RK, Yu M, Weleber RG. Multifocal electroretinographic evaluation of long-term hydroxychloroquine users. Arch Ophthalmol Chic III 1960 2004; 122: 973-981

[21] Sebastiani S, Fresina M, Cellini M et al. Hydroxychloroquine for treatment of rheumatoid arthritis: multifocal electroretinogram and laser flare-cell photometry study. Clin Ophthalmol AuckI NZ 2017; 11: 689-696

[22] Xiaoyun MA, Dongyi HE, Linping HE. Assessing chloroquine toxicity in RA patients using retinal nerve fibre layer thickness, multifocal electroretinography and visual field test. $\mathrm{Br}$ J Ophthalmol 2010; 94: 1632-1636

[23] Ahn S], Ryu S], Lim HW et al. Toxic effects of hydroxychloroquine on the choroid: Evidence From Multimodal Imaging. Retina Phila Pa 2019; 39: 1016-1026

[24] Miro O, Pedrol E, Casademont J et al. Muscle involvement in rheumatoid arthritis: clinicopathological study of 21 symptomatic cases. Semin Arthritis Rheum 1996; 25: 421-428

[25] Tselios K, Deeb M, Gladman DD et al. Antimalarial-induced Cardiomyopathy in Systemic Lupus Erythematosus: As Rare as Considered? J Rheumatol 2019; 46: 391-396

[26] Chatrel C, Roubille F, Vernhet $\mathrm{H}$ et al. Cardiac Complications Attributed to Chloroquine and Hydroxychloroquine: A Systematic Review of the Literature. Drug Saf 2018; 41: 919-931

[27] Costedoat-Chalumeau N, Hulot J-S, Amoura Z et al. Heart conduction disorders related to antimalarials toxicity: an analysis of electrocardiograms in 85 patients treated with hydroxychloroquine for connective tissue diseases. Rheumatology (Oxford) 2007; 46: 808-810

[28] Götestam Skorpen C, Hoeltzenbein M et al The EULAR points to consider for use of antirheumatic drugs before pregnancy, and during pregnancy and lactation. Annals of the Rheumatic Diseases 2016; 75: 795-810

[29] Klinger G, Morad Y, Westall CA et al. Ocular toxicity and antenatal exposure to chloroquine or hydroxychloroquine for rheumatic diseases. Lancet 2001; 358: 813-814

[30] Motta M, Tincani A, Faden D et al. Follow-up of infants exposed to hydroxychloroquine given to mothers during pregnancy and lactation. J Perinatol 2005; 25: 86-89

[31] Marmor MF, Kellner U, Lai TYY et al. American Academy of Ophthalmology. Recommendations on Screening for Chloroquine and Hydroxychloroquine Retinopathy (2016 Revision). Ophthalmology 2016; 123: 138694

[32] Opthalmologists TRC of. Clinical Guidelines [Internet]. R. Coll. Ophthalmol. [zitiert 30. Dezember 2018]. Verfügbar unter https:// www.rcophth.ac.uk/standards-publications-research/clinical-guidelines/ 